\title{
Rainfall Prediction using Genetic Algorithm
}

\author{
J.Refonaa, M. Lakshmi, R S S SrinivasaRao, P Eshwar Prasad
}

\begin{abstract}
Numerousresearch works are being carried out by various people to predict the occurrence of rainfall before it actually comes so as to minimize the amount of damage to a particular area or make the people of the area aware of the rain hit so that they could take some previous safety measures. Various classifiers and artificial neural networks are preferred by various researchers to predict the rain in a given geographic area. Use of genetic algorithms have seen in intense use for various research purposes and prediction of weather is not an exception. In this paper, we have proposed a system that could predict the rainbeforehand using genetic algorithm. Matlab is used for observing the performance of the algorithm. Genetic algorithm is used for feature extraction from the input dataset. When compared to other approaches use of genetic algorithms seems s to be more efficient for predicting the rain as hence it is used in the current work. The evaluation results are performed based on evaluating various parameters and the proposed model seems to provide a better efficiency when compared to the rest of the previous traditional rainfall prediction systems.
\end{abstract}

Keywords - Rainfall Prediction, Genetic Algorithm, Crossover, Mutation, Accuracy, Evaluation, Fitness

\section{INTRODUCTION}

Rainfall is stated as one among the most dangerous hazards that could cause severe damages to numerous parts of the country or a given geographic area especially in the parts of India, China, Bangladesh,and Taiwan. These floods majorly contribute to almost seventy fivepercent of risk of death around the world and it also plays a major role in about two million folks which are full of impact including the mountain landslide. Apart from this rainfall is also responsible for about seventy-eight million folks of exposing in an annum that lies inside the boundaries of a dangerous tropical cyclone. Extraordinarily most of the very dangerous hazards in the country occur due to the frequent rainfall in our country and hence it suffers from numerous disasters.

As there are numerous technologies that are been vitally used for many of the research purposes, detection of weather is not an exception[11,12,13]. Many research works are pertaining to predicting the climatic conditions well before to minimize the amount of damage to lives and properties. Rainfallis one of the most important climatic change that needs to be predicted well before as our country is losing numerous lives due to this and alsodue to the disaster that is caused due to heavy rainfall.

In this paper, we have proposed a system that makes use of a genetic algorithm for extracting the features of the dataset. The genetic algorithm is also used for finding the accuracy and performance of the entire proposed model. The

Revised Manuscript Received on July 10, 2019.

J.Refonaa, Assistant Professor, School of Computing, Sathyabama Institute of Science and Technology. Chennai, T.N, India.

Dr. M. Lakshmi, Principal, Professor, Sri krishna College of Technology, Coimbatore, T.N, India.

R S S SrinivasaRao, Student, School of Computing, Sathyabama Institute of Science and Technology, Chennai, T.N, India.

P Eshwar Prasad rest of the section is as follows: Section II consists of Literature Survey, section III consists of the methodology used in the paper and section III consists of various results obtained. The paper is concluded in the last by mentioning the relevant future works that could be applied or added to the proposed work.

\section{RELATED WORK}

There are numerous works that have been proposed by various researchers for predicting the rainfall in a given geographic area. In [1], the author has performed a typical survey on all the available Neural Network architectures that are intensely used for predicting the rainfall in the last twenty-five years. The authors highlighted that almost all of the researchers got vital leads to downfall prediction by exploitation Propagation Network, furthermore, the statement techniques that used SVM, MLP, BPN, RBFN, and Kyrgyzstani monetary unit are additional appropriate than alternatively applied math and numerical techniques. Some limitations have additionally been highlighted. Researchers in [2] used prediction in the Asian country. For future direction, it absolutely was urged that few extra options would be enclosed in a computer file for downfall prediction like ocean Surface Temperature for the areas around Andhra Pradesh and Southern a part of India. Researchers in [3] foretold monthly downfall by exploitation Back Propagation, Radial Basis performs and Neural Network. For prediction, the dataset was collected from district Tamil Nadu. Researchers in [4] bestowed a Hybrid System. Researchers in [5] mentioned downfall pace in previous years with relation to varied crops seasons like rabi, Kharif, said then foretold (rainfall) for future seasons. In [6], one month and 2-month statement models were developed for downfall prediction. The input dataset was selected from multiple stations in North India, spanned on past 141 years. Neural Network exploitation Back Propagation and Levenberg-Marquardt coaching perform were employed in these models. Researchers in [7] bestowed associate degree formula by integration of data processing and applied math Techniques. Indian Ocean dipole conditions and southern oscillation were taken In [8], the researchers have predicted the rate of rainfall by using the Wave Neural Network (WNN), that is associated with the degree of integration odsome of the commonly used wave techniques that are inturn used for investigating the overall performance. In $[9,10]$, the researchers have proposed a generic survey and have also performed an overall analysis of assorted neural networks on predicting the rainfall of the particular area. Most of the research work proposed consumes a high computation time while giving a comparatively low efficiency.

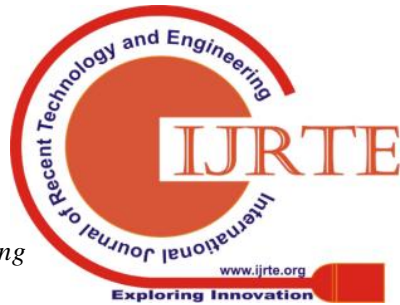




\section{PROPOSED MODEL}

Themodel that is proposed in the present paper is as shown in Fig. 1. The block diagram is depicted which shows the genetic algorithm that is intensely used in feature selection. The feature selected from the input dataset is used with the selected feature. The metrics are used to calculate the accuracy of the proposed model. The initial dataset is used to train the model for predicting the rainfall model.

Data processing is done for the given input dataset. The classification of the dataset is done using the back propagation method. The model consists of three modules. The first one is the Preprocessing module where the input is taken and then converted for extracting the features. The second one is the Genetic Algorithm. Popular functions such as crossover and mutation operators are used for predicting the rainfall. The final module is the Classifier where the training is given and when a new data is given it is able to predict the classification based on previous training. The prediction is done for measuring the accuracy of the model. The data that is obtained is unique when compared to other models. The output that is generated is the prediction of the rainfall values for predicting the final output values.

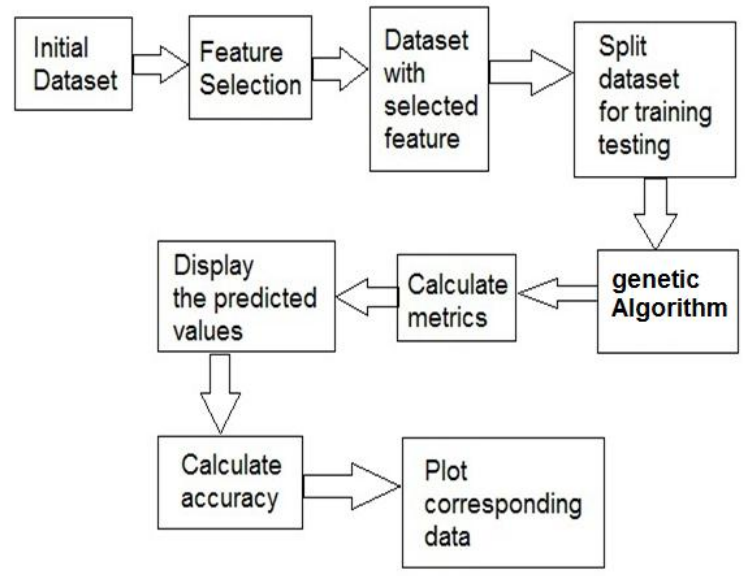

Fig. 1 Block Diagram of Proposed Prediction Model

The entire architecture diagram of the proposed model is shown in Fig. 2. The parameters are used to be given as the input for the model.

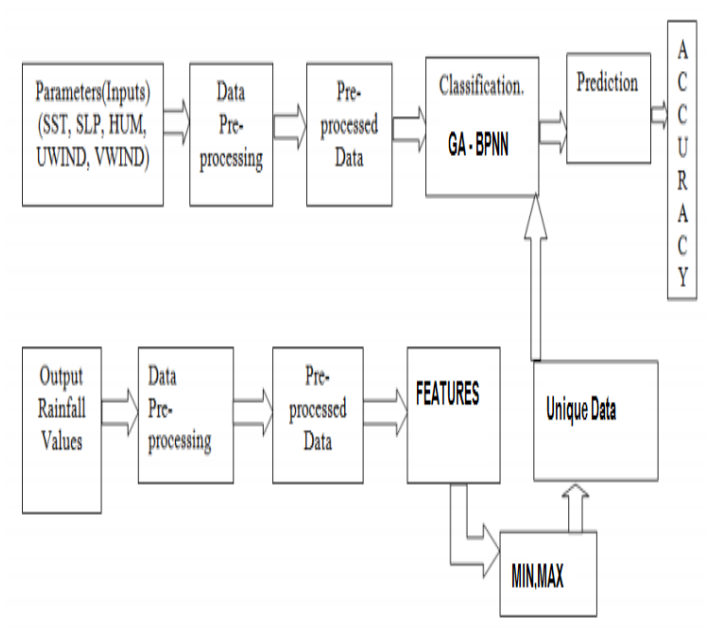

Fig. 2 Architecture of Proposed Prediction Model

\section{Genetic Algorithm}

In Fig.3, we have a flow of genetic algorithm. It will assign the fitness using the selection, crossover and mutation operators. Then if the value is true then it will go for another prediction. If it is false then again it will go for assigning the fitness using this three operator. All this three operator has unique operations. This algorithm was used to predict the rainfall dataset values. For choosing best from the selected region or area. It was helped our proposed system to predict the values in faster and giving the best performance validation.

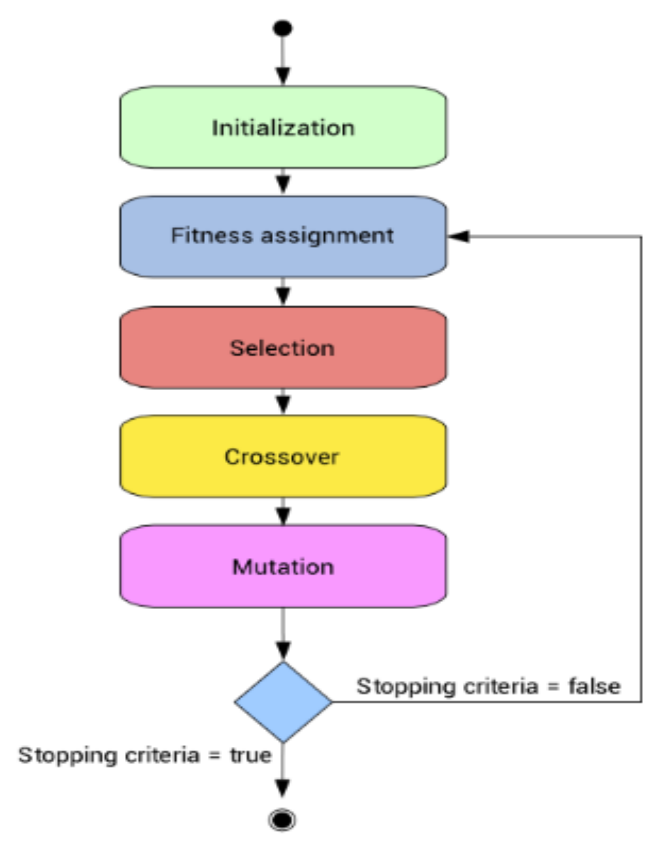

Fig. 3Genetic flow

\section{Selection operator}

This operator will choose the best out of all. Selecting the good fitness from the population. This operation will suite for predicting the rainfall dataset values to select the best area in the data. This operator was used in our system to predict the individual values by using the scheme. This is the first scheme in the genetic algorithm

\section{Crossover operator}

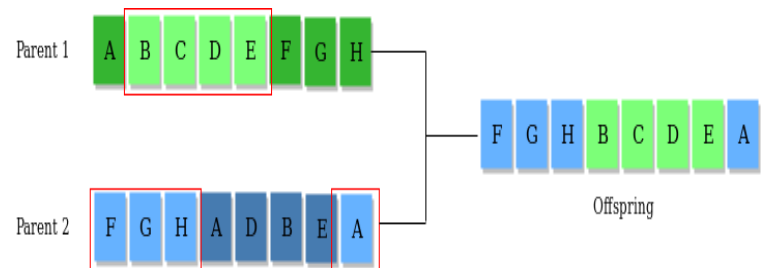

Fig. 4Crossover operator

Crossover operation will mating the two individuals to create the good values. In Fig.4, there two parents, each are good individuals value. By mating this two values, this operator will create the new individual by mating the two good values to produce one offspring. Using this operation, 
we can predict the rainfall values with different datasets. It will also use to compare the best values out of two individuals. We have fixed this operation as second option in our proposed system, it was satisfied many areas while testing.

\section{Mutation operator}

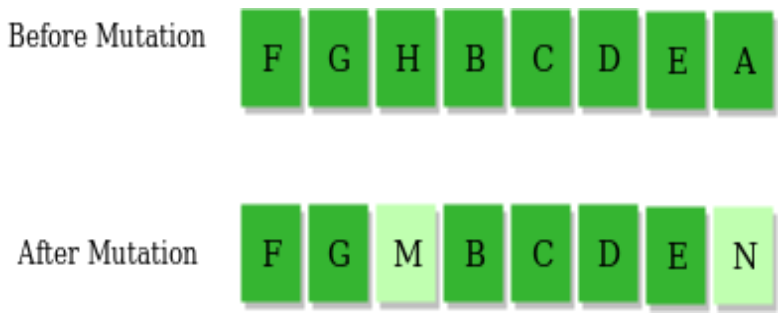

Fig. 5Mutation process

Mutation operator will insert the random values to the individuals for avoiding the diversity. Hence this scheme is proved the system very well and organized it many ways. We have proposed all these three schemes for predicting the best values from the dataset and the rainfall region. If we use the engine to predict the particular area, then mutation will set some values for previous predicted values for picking up the same data in later. We have discussed our result in the next section with some screeshots.

\section{EXPERIMENTAL RESULTS}

The experimental results are obtained using the Matlab software. The input dataset isfirst given to the classifier to obtain the accuracy of the entire model. In Fig. 6, the results obtained for predicting the rainfall is shown. The output target gives the rainfall that has been identified in the previous years of a given region. Here we have analyzed the best output by seeing the response time for series 1 , then we have displayed the error area in the graph representation. So using this values of dataset. We can predict the rainfall in accurate and good response.
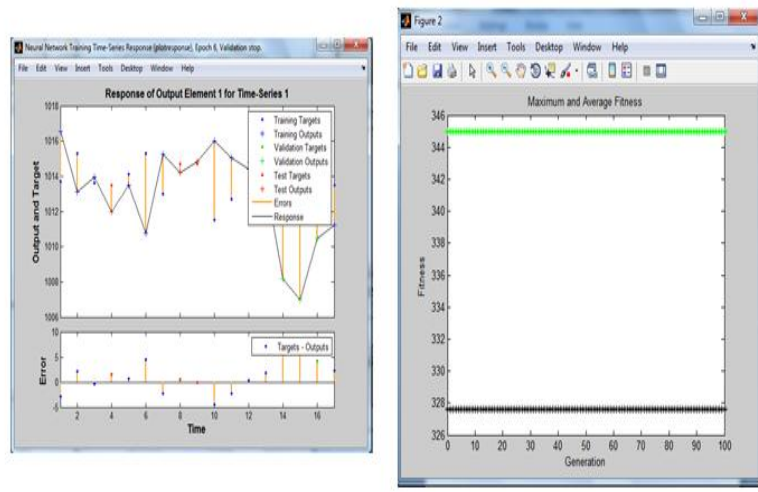

Fig. 6Result Evaluation for Rainfall Prediction

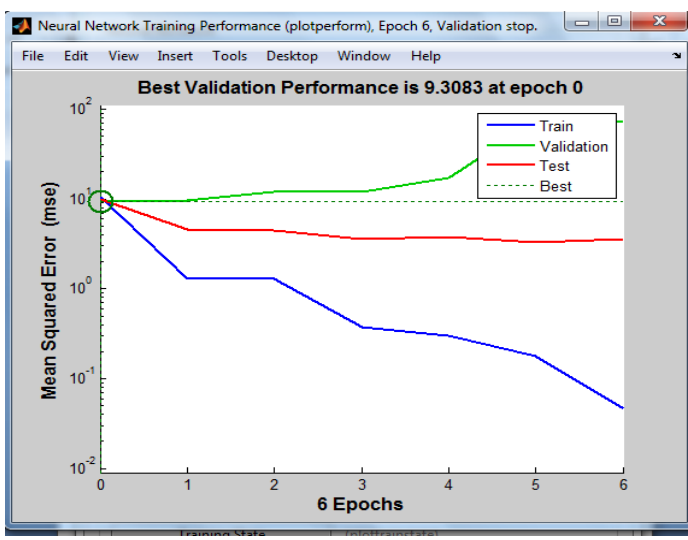

Fig. 7 Best performance

When we look into Fig.7, it is shown the prediction of rainfall in India with best performance validation. The model is able to predict the rainfall with better efficiency. In Fig.8, we can see the autocorrelation of error was set to limit for seeing the confidence and the prediction values in the best performance. Bode diagram will show the division point for in depth analysis. After inserting the rainfall dataset into our system, it will firstly show the response, error, performance analysis, autocorrelation and then bode diagram. Using this steps, we can collect the values from all scheme and building it as division type of graph.

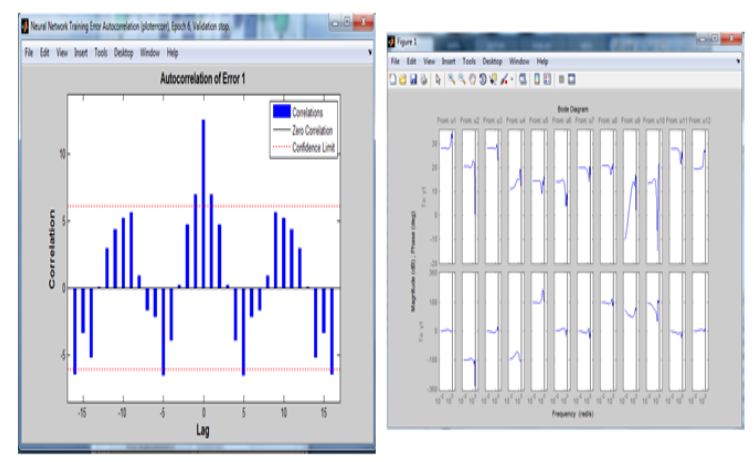

Fig. 8 Autocorrelation of error and bode diagram

\section{CONCLUSION}

There are numerous technologies that are growing with the growth in the technical era. Prediction of weather has intensively got a rapid growth in the imminent technological era. Genetic algorithms play a vital role in classification techniques and it also plays a vital role in predicting the weather of a specific area or a country for a particular period of time. In this paper, we have proposed an efficient model of using a genetic algorithm for predicting the rainfall in a particular area well before to avoid the loss of lives and property damage. The model proposed using genetic algorithm extracts the features from the given dataset. The proposed model is observed to perform better than any of the previously existing models. Future work could include the use of any security protocols for securing the entire system.

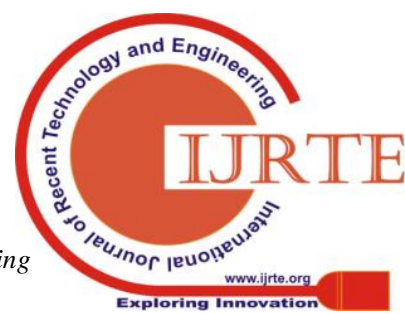




\section{REFERENCES}

1. D. Nayak, A. Mahapatra, and P. Mishra, "A Survey on Rainfall Prediction using Artificial Neural Network," Int. J. Comput. ..., vol. 72, no. 16, pp. 32-40, 2013.

2. B. K. Rani and A. Govardhan, "RAINFALL PREDICTION U SING DATA MINING TECHNIQUES - A SURVEY," pp. 23-30, 2013.

3. N. Tyagi and A. Kumar, "Comparative analysis of backpropagation and RBF neural network on monthly rainfall prediction," Proc. Int. Conf. Inven. Comput. Technol. ICICT 2016, vol. 1, 2017

4. N. Solanki and G. P. B, "A Novel Machine Learning Based Approach for Rainfall Prediction," Inf. Commun. Technol. Intell. Syst. (ICTIS 2017) - Vol. 1, vol. 83, no. Ictis 2017, 2018.

5. M. Ahmad, S. Aftab, and I. Ali, "Sentiment Analysis of Tweets using SVM," Int. J. Comput. Appl., vol. 177, no. 5, pp. $25-29,2017$

6. C. S. Thirumalai, "Heuristic Prediction of Rainfall Using Machine Learning Techniques," no. May, 2017.

7. N. Mishra, H. K. Soni, S. Sharma, and A. K. Upadhyay, "Development and Analysis of Artificial Neural Network Models for Rainfall Prediction by Using Time-Series Data," Int. J. Intell. Syst. Appl., vol. 10, no. 1, pp. 16-23, 2018.

8. H. Vathsala and S. G. Koolagudi, "Prediction model for peninsular Indian summer monsoon rainfall using data mining and statistical approaches," Comput. Geosci., vol. 98, pp. 5563, 2017.

9. R. Venkata Ramana, B. Krishna, S. R. Kumar, and N. G. Pandey, "Monthly Rainfall Prediction Using Wavelet Neural Network Analysis," Water Resour. Manag., vol. 27, no. 10, pp. 3697-3711, 2013.

10. Rajalakshmi, V., M. Lakshmi, and V. Maria Anu. \&quot;A Complete Privacy Preservation System for Data Mining Using Function Approximation.\&quot; J. Web Eng. 16, no. 3\&amp;4 (2017): 278-293.

11. S.Ancy, Kumar. R, Asokan.R ,Subhashini.R, "Prediction of Onset of South West Monsoon using Multiple Regression"On "Image Processing" at the "2nd IEEE- International Conference on Computer Communication and Systems" (ICCCS 2014) on at Saveetha Engineering College, Chennai on 20th \& 21st February, 2014. ISBN No: 978-1-4799-3671-7

12. Sethuraman, R., Chiranjeevi Saagar, K.B "Importing adaptive database schema design" International Journal of Applied Engineering Research Volume 10, Number 20 (2015) pp.18490-18493.

13. Saravanan, M, Clustering of data sets by using fuzzy algorithm, ARPN Vol 10, 2015 